\title{
L'innovation sociale dans des espaces fragmentés entre rationalité en valeur et rationalité instrumentale. Le cas des démences dans les soins à domicile en Allemagne et en Suisse
}

Social innovations in fragmented contexts between value rationality to instrumental rationality. Dementia Domiciliary Long-Term care in Germany and Switzerland

\section{Olivier Giraud et Barbara Lucas}

\section{(2) OpenEdition}

\section{Journals}

Édition électronique

URL : https://journals.openedition.org/pmp/11462

ISSN : 2119-4831

Éditeur

Institut de Management Public (IDPM)

\section{Édition imprimée}

Date de publication : 1 janvier 2018

Pagination : 69-86

ISBN : 978-2-7430-2415-4

ISSN : 0758-1726

\section{Référence électronique}

Olivier Giraud et Barbara Lucas, « L'innovation sociale dans des espaces fragmentés entre rationalité en valeur et rationalité instrumentale. Le cas des démences dans les soins à domicile en Allemagne et en Suisse », Politiques et management public [En ligne], Vol 35/1-2 | 2018, mis en ligne le 12 novembre 2021, consulté le 15 novembre 2021. URL : http://journals.openedition.org/pmp/11462 


\title{
L'innovation sociale dans des espaces fragmentés entre rationalité en valeur et rationalité instrumentale Le cas des démences dans les soins à domicile en Allemagne et en Suisse
}

\author{
- Olivier Giraud ${ }^{a *}$ et Barbara Lucas ${ }^{b}$ \\ ${ }^{a}$ Lise-CNRS-Cnam, Paris \\ ${ }^{b}$ HETS-HES-SO, Genève
}

\section{Résumé}

La plupart des analyses disponibles dans la littérature font état des blocages générés par les situations de segmentation dans l'action publique. Cet article, basé sur deux études de cas d'innovations locales dans le domaine de l'aide et des soins à domicile des personnes atteintes de démence, situées dans des pays fédéraux (Allemagne et Suisse), montre que deux mécanismes permettent de surmonter les segmentations. Des coalitions portant sur le contenu même de l'innovation sociale avec d'autres acteurs (rationalité en valeur) se combinent avec des stratégies d'acquisition des ressources nécessaires à la bonne marche des innovations (rationalité instrumentale) pour surmonter les segmentations institutionnelles. Sur la base des succès plus ou moins grands des trajectoires d'innovation dans les deux études de cas retenues, il est possible de conclure sur les conditions de combinaison positive entre les deux logiques mises en évidence.

(c) 2018 IDMP/Lavoisier SAS. Tous droits réservés

Mots clés : innovation sociale, comparaison, instruments d'action publique, systèmes locaux d'aide et de soins à domicile, analyses multi-scalaires.

\section{Abstract}

Social innovations in fragmented contexts between value rationality to instrumental rationality. Dementia Domiciliary Long-Term care in Germany and Switzerland. The prevailing literature about segmentation in policy settings associates those ruptures with blockages. This article, based on two local case studies in the policy domain of domiciliary dementia long-term care in two federal countries (Germany and Switzerland), shows that two specific mechanisms can overcome segmentations. Actors' coalitions about the very content of social innovation (value rationality) as well as strategies aimed at acquiring the necessary resources in the context of the trajectory of social innovation (instrumental rationality) may overcome

*Auteur correspondant : olivier.giraud@cnam.fr

doi :10.3166/pmp.35. 2018.0004 @ 2018 IDMP/Lavoisier SAS. Tous droits réservés 
those blockages. From both the successes and the shortcomings of both local case studies of social innovation trajectories we explore the conditions for a positive combination of both logics.

(c) 2018 IDMP/Lavoisier SAS. Tous droits réservés

Keywords : social innovation, comparison, policy instruments, local domiciliary long-term care systems, multiscalar analysis.

\section{Introduction}

Cet article traite des mécanismes sociaux rendant possible l'innovation sociale dans des contextes d'action publique fragmentés, le plus souvent considérés comme peu favorables à toute forme de changement. Nous nous intéressons plus particulièrement aux trajectoires de deux innovations locales dans le domaine de l'aide et des soins à domicile pour les personnes atteintes de démences. La première innovation est située en Suisse, dans le canton de Vaud. Elle vise à offrir du répit aux proches de personnes souffrant de démence, en venant les relayer à domicile. Le projet, intitulé les Alzamis est initié à petite échelle par une association locale. La seconde innovation a été lancée par la municipalité de Ahlen, une ville allemande de Westphalie orientale. Elle a pour objectif de favoriser le recours des personnes migrantes et atteintes de démence et de leurs proches aux services d'aide et de soins à domicile. Si ces deux innovations ont obtenu une certaine institutionnalisation, leurs trajectoires sont contrastées. En effet, les Alzamis ont bénéficié d'un soutien important des services du canton de Vaud et sont parvenus à concrétiser une offre de services originale et importante pour les acteurs locaux. Par contraste, l'initiative de Ahlen, pourtant portée par la municipalité, n'a pas réussi, au moins pendant la période de notre observation empirique, à lier les réseaux sociaux des populations issues de l'immigration et ceux des prestataires d'aide et de soins à domicile.

Le contraste de ces trajectoires nous confronte à une question classique de l'analyse des politiques publiques : celle des liens entre « changement institutionnel » et « changement réel » (Streeck, Thelen, 2005). Par changement réel, nous entendons un changement qui aboutit à une transformation des pratiques sociales dans un domaine spécifique. Cette caractérisation correspond en effet à la définition aujourd'hui dominante de l'innovation dite sociale: "An innovation is therefore social to the extent that it, conveyed by the market or "non/without profit", is socially accepted and diffused widely throughout society or in certain societal sub-areas, transformed depending on circumstances and ultimately institutionalized as new social practice or made routine.» (Howaldt, Schwarz, 2010 : 21). Pour rendre compte des trajectoires dans laquelle l'innovation aboutit - ou non- à un changement réel, nous pensons que celle-ci doit être analysée dans le cadre de la politique publique qui régule son domaine d'intervention, en portant une attention particulière aux enjeux de coordination entre les acteurs du réseau d'action publique.

Comme le soulignent Rose et Davies, une politique publique se présente comme une « combinaison spécifique de lois, d'affectations de crédits, d'administrations et de personnels dirigés vers un ensemble d'objectifs plus ou moins clairement définis » (Rose et Davies 1994 : 54). Chacun de ces acteurs - administration, personnel - comme de ces étapes - lois, crédits ou autres - sont insérés dans des contextes institutionnels et sectoriels 
(professionnels par exemple) bien différenciés, ce qui suppose d'intenses coordinations ou accords entre ces différents acteurs. Dans le processus d'action publique, ces coordinations portent sur deux enjeux précis. Le premier est celui du rapport aux finalités qui structurent l'action publique. Ainsi, les objectifs qualitatifs de l'innovation représentent une opportunité de forger des accords, voire des coalitions entre acteurs. Lutter contre l'isolement des personnes âgées à mobilité réduite est un exemple d'un tel objectif qualitatif. Le second enjeu de coordination concerne la mobilisation et la combinaison des ressources dont disposent les différents acteurs dans le but de produire une action spécifique. Il suppose pour eux de partager une appréciation sur l'efficacité d'un dispositif et des ressources qu'ils peuvent mettre au service de l'objectif qualitatif. Les accords sur les aspects instrumentaux de l'innovation sont au moins aussi importants que ceux construits sur les finalités car ce sont eux qui guideront la mise en œuvre de l'innovation, en forgeront ainsi la matérialité, et par là même, détermineront les styles d'interaction entre prestataires et destinataires, comme l'intensité de la mise en œuvre (Halpern, Lascoumes, Le Galès, 2014).

Dans le cas de l'aide et les soins à domicile en Suisse et en Allemagne, la fragmentation est particulièrement importante. Le domaine se caractérise par une forte segmentation institutionnelle entre secteurs sanitaire et social, compétences fédérales, régionales (Länder et cantons) et locales, mais aussi entre des acteurs professionnels et des destinataires dont les valeurs et les attentes se pluralisent. La littérature sur le changement institutionnel et les innovations assimile souvent de tels contextes à la multiplication des blocages (Duran, 2010) et à des coûts de transaction élevés (Hooghe et Marks, 2003). Nos observations empiriques montrent toutefois que des acteurs peuvent développer des stratégies efficaces pour dépasser la fragmentation institutionnelle, entre échelles d'action, mais aussi entre types d'acteurs. De façon plus précise, plutôt que de parler de façon générique « d'action collective positive » (Benz, 2009), nous pensons qu'une approche théorique fondée sur les coalitions discursives permet d'identifier la façon dont les acteurs qui portent l'innovation sociale sont capables de dépasser les segmentations institutionnelles, professionnelles, organisationnelles.

L'analyse des aspects discursifs de l'action publique, notamment selon l'approche proposée par Marteen Hajer (1997), permet en effet de comprendre les logiques d'élargissement des coalitions d'acteurs impliqués dans un projet par l'élaboration d'un narratif commun et son inscription dans des pratiques institutionnalisées. Ce processus engage le contenu même de l'innovation, et les valeurs sur lesquelles elle se fonde, mais aussi la compréhension qu'ont les acteurs des façons de mobiliser les ressources et moyens nécessaires ou qu'ils ont à disposition, selon deux rationalités distinctes et à première vue opposée. D'un côté, les acteurs formulent collectivement les objectifs de l'innovation en cherchant chacun à défendre un ensemble de normes et de valeurs qui leur sont propres. L'enjeu pour les porteurs de l'innovation est ici d'en élargir la formulation initiale aux éléments de discours proposés par les acteurs qui rejoignent la coalition sans remettre cause les valeurs qui sont au cœur du projet. D'un autre côté, le comportement des acteurs est motivé par des jeux stratégiques appliqués à la préfiguration des formes de mise en œuvre de l'innovation. L'enjeu pour les porteurs du projet est ici de négocier les ressources nécessaires à sa réalisation. Or, la mobilisation conjointe de ces deux rationalités se révèle comme un élément crucial dans la réalisation d'une innovation. Comme l'illustrent les destins contrastés des trajectoires d'innovation dans nos cas suisses et allemands, seule l'ouverture du discours innovant vers 
les positions d'autres acteurs, ainsi que la construction d'une logique de mobilisation des ressources crédible permet au final de déboucher sur du changement social, c'est-à-dire sur la concrétisation de l'innovation par son adoption par ses destinataires potentiels.

La suite de cette contribution s'organise en quatre parties. Dans une première partie, nous revenons, d'un point de vue théorique, sur les enjeux spécifiques de l'innovation sociale dans un contexte de forte fragmentation organisationnelle, institutionnelle et politique et proposons un cadre d'analyse centré sur la construction des coalitions discursives. La deuxième partie, méthodologique, présente la recherche dont sont tirés les deux cas et résume la méthode mobilisée. Dans la troisième partie, nous présentons l'analyse des deux trajectoires d'innovation sociale à Ahlen (Allemagne) et dans le canton de Vaud (Suisse). Nous nous intéressons d'abord à la constitution de coalitions discursives entre les initiateurs de l'innovation et d'autres acteurs autour de la définition des objectifs du projet, puis nous analysons la manière dont l'institutionnalisation de ces deux innovations engage de façon différenciée la mobilisation de ressources par les porteurs de l'innovation. Enfin, dans une partie conclusive, nous revenons sur les mécanismes de l'innovation sociale en régime d'action publique fragmenté. Nous soutenons qu'une innovation capable de produire des effets sociaux dans le contexte d'une politique publique locale implique la maîtrise conjointe de deux formes de rationalités apparemment opposée : une rationalité en valeur et une rationalité instrumentale.

\section{Cadre théorique}

\subsection{La fragmentation comme frein à l'innovation sociale}

Dans un article récent, Stephen Sinclair et Simone Baglioni définissent l'innovation sociale comme « une réponse spécifique et efficiente à des besoins non pris en compte et qui poursuit des objectifs sociaux visant à augmenter les ressources sociales et les capacités [des individus] » (Sinclair, Baglioni, $2014:$ 471). Cette définition valorise la contribution sociale positive de l'innovation du point de vue des individus, mais aussi de la collectivité. Se concentrant cependant sur les effets de l'innovation sociale, elle laisse dans l'ombre les aspects qui touchent, si ce n'est aux «causes », au moins aux mécanismes et aux conditions qui rendent possibles l'innovation sociale. Or, si cette interrogation nous semble fondamentale, elle ne saurait, comme un grand nombre de travaux le suggèrent, assimiler l'innovation sociale à des apprentissages sociaux ancrés dans la gouvernance des enjeux sociaux concernés (pour des recensions de ces approches : Ewert, Evers, 2014; Jenson, Harrisson, 2013).

Une définition de l'innovation sociale diffusée par l'Union Européenne lie le contenu des services ou prestations innovantes à des réseaux sociaux et relations sociales qui l'ont portée ou bien qu'elle alimente. Elle renvoie alors à des « idées nouvelles qui non seulement rencontrent des besoins sociaux mais aussi créent de nouvelles relations sociales » (BEPA, 2010). Cette perspective inclut les différents acteurs concernés par le processus d'innovation dans un domaine spécifique que cela soit à travers la définition des besoins, la conception des réponses, la mise en œuvre des dispositifs ou encore l'usage de ces dispositifs. Cette vision inclusive des relations sociales outre le fait qu'elle permet de conserver la valeur 
ajoutée de la notion d'innovation sociale, par rapport à des notions proches comme celle de changement ou apprentissage, est utile dans un domaine particulièrement segmenté comme l'aide et les soins à domicile des personnes âgées en perte d'autonomie. Plus encore, cette définition est compatible avec celle, évoquée en introduction à cet article, et qui fait le choix d'assimiler la dimension « sociale » de l'innovation sociale à la thématique du changement social (Howaldt, Schwarz, $2010: 21$ ).

La diffusion d'une innovation dans un domaine d'action publique segmenté comme le nôtre, situé à l'articulation entre les secteurs de la santé et du social, pose des problèmes spécifiques de coordination d'institutions administratives, de flux financiers, mais aussi de réseaux d'acteurs pris dans des fonctionnements et des allégeances propres. Cette segmentation horizontale est par ailleurs renforcée par le caractère pluraliste des acteurs impliqués - publics, marchands, associatifs- et plus encore, par leurs ancrages dans des échelles d'action publique diverses. L'enrôlement croissant des proches des bénéficiaires dans les dispositifs d'action publique (Giraud, Lucas, 2015b), notamment via le développement de mesures d'indemnisation financière (Le Bihan, 2011), est un enjeu de fragmentation supplémentaire. Enfin, la pluralisation des services et prestations liée à celle des besoins, cultures, modes de vie, solidarités familiales ou de proximité des bénéficiaires d'aide et de soins à domicile comme de leurs proches constitue un enjeu de plus en plus important de différenciation des acteurs et des services dans ces secteurs.

Ces contextes fragmentés ont conduit des chercheurs à développer la notion « d'espace fonctionnel de régulation » (Functional Regulatory Space) (Varone et al., 2013), dans le cas d'autres domaines d'action publique, selon laquelle de tels espaces, qui n'ont pas de base institutionnelle ou organisationnelle propre, existent, de façon relativement autonome, dans le but de « traiter, appuyer ou résoudre des problèmes qui concernent différents secteurs d'action publique dans des territoires institutionnels variés et inscrits à plusieurs échelles de gouvernement » (Ibid., p. 319). Le traitement concret des problèmes publics, comme leur conception et formulation sont concernées. Comme Patrice Duran le souligne, la plupart des problèmes publics dépassent aujourd'hui les frontières administratives des « secteurs » d'intervention de l'État. Il en conclut qu'il est nécessaire pour les structures publiques de procéder aussi bien à des intégrations verticales qu'horizontales pour pouvoir s'adapter de façon optimale (Duran, 1999, 2010, : 145 ; Bezès, Le Lidec, 2016).

Dans l'ensemble, la littérature sur le changement institutionnel et les innovations assimile souvent de tels contextes institutionnels fortement segmentés à la multiplication des blocages (Duran, 2010) et à des coûts de transaction élevés (Hooghe et Marks, 2003). Ce point de vue est-il pour autant indépassable? N'existe-t-il pas des analyses, peut-être plus réalistes, qui considèrent les systèmes d'action publique tels qu'ils sont, et s'attachent à montrer leurs capacités de fonctionnement, voire d'innovation, en dépit, voire le cas échéant, grâce à certaines des segmentations qui caractérisent ces domaines?

\subsection{Dépasser la fragmentation - la construction de coalitions discursives}

Bien que la littérature dominante assimile de manière générale la fragmentation de l'action publique à un risque de fonctionnement déficient, on trouve également des interprétations de la fragmentation comme opportunités pour l'innovation sociale. Cet enjeu est particulièrement important dans des pays fédéraux marqués par une fragmentation verticale 
- plus encore pour la Suisse que pour l'Allemagne - mais aussi par le poids traditionnel important des acteurs privés, notamment associatifs, dans le pilotage et la mise en œuvre de l'action publique, en particulier dans le champ des politiques sociales.

Pour saisir la façon dont une innovation sociale peut dépasser ce type de fragmentation généralisée, voire se hisser au niveau d'une remise en question des valeurs qui sous-tendent la politique mise en œuvre, alors même que les narratifs scalaires libéraux tendent à rabattre l'innovation locale au seul ajustement aux contraintes économiques - nous reprenons l'idée des coalitions discursives mises en avant par Maarten Hajer (1997). Selon cet auteur, «a discourse coalition is the ensemble of a set of story lines, the actors that utter these story lines, and the practices that conform to these story lines, all organized around a discourse ». (Hajer 1993 : 47). Deux éléments clé sont inclus dans cette définition. En premier lieu, des acteurs appartenant à des univers différents peuvent conclure des coalitions du fait qu'ils partagent un même discours à propos d'un enjeu d'action publique. En second lieu, ces discours renvoient à des pratiques, auxquelles ils donnent sens et induisent le changement social, en lui-même directement lié à l'innovation sociale.

La conclusion de coalitions autour d'un même discours (narrative storylines) entre acteurs dont les préférences, modes de fonctionnement, appartenances institutionnelles à des réseaux sont différents est essentielle pour comprendre le dépassement de la fragmentation de l'espace d'action, et partant, pour comprendre la dynamique de l'innovation sociale. Les narratifs concernés portent sur les finalités en valeurs de l'innovation sociale, mais aussi sur les moyens adéquats de la mettre en œuvre.

Pour ce qui concerne les finalités, le discours qui sous-tend l'innovation doit être élargi, au moins partiellement, aux positions des acteurs qui entrent dans la coalition, de manière à ce que ces acteurs deviennent véritablement actifs au bénéfice de la réalisation de l'innovation sociale. Par exemple, une innovation sociale proposant d'introduire une démarche qualité dans les prestations d'aide et de soins à domicile destinées aux personnes âgées d'une commune pourra d'autant plus facilement recevoir le soutien des associations des professionnels du domaine, que les normes de professionnalité seront reconnues comme un fondement important à la démarche qualité en question. La dynamique des coalitions discursives aboutit ainsi à reformuler en partie le contenu originel de l'innovation sociale (Giraud et al., 2014). Dans l'exemple proposé ici, la démarche qualité devient plus spécifiquement une démarche qualité fondée sur des normes professionnelles.

Les ressources ou moyens à mobiliser pour la réalisation de l'innovation en question jouent également un rôle crucial dans la constitution des coalitions discursives. Selon Hajer, une coalition discursive peut être considérée comme dominante dans un champ politique donné lorsqu'elle parvient à la fois à structurer l'espace discursif, à savoir lorsque des acteurs centraux sont convaincus ou acceptent de partager le nouveau discours et lorsque le discours se traduit dans des arrangements institutionnels. Dans le cas de notre exemple d'une innovation portant sur l'introduction d'une démarche qualité dans un système local d'aide et de soins à domicile, on peut penser à différents instruments qui organisent la mobilisation des ressources par les acteurs impliqués, qui induiront des pratiques institutionnalisées contrastées pour les acteurs. Deux exemples, assez polarisés, illustrent la diversité des options envisageables : un code de bonne conduite volontaire diffusé auprès des prestataires locaux par une association locale ; l'intégration de normes qualité strictes aux contrats de prestation signés par la commune. 


\section{Méthode : la comparaison de deux innovations locales en Allemagne et en Suisse}

Le but de notre contribution est d'identifier, sur la base de deux études de cas contrastés, les mécanismes sociaux qui permettent l'institutionnalisation d'une innovation dans un contexte institutionnel segmenté. Nous comparons les trajectoires de ces deux innovations sociales dans le domaine de l'aide et des soins aux malades d'Alzheimer. Ces deux cas sont tirés d'une recherche récente portant sur l'innovation sociale dans le domaine de la prise en charge des démences faisant elle-même suite à un premier programme de recherche concernant les personnes âgées en perte d'autonomie en général ${ }^{1}$. Le design de recherche des deux projets était similaire et les trois cas nationaux - Allemagne, Écosse, Suisse - étaient les mêmes. Ce domaine a été choisi en raison de sa forte fragmentation institutionnelle, professionnelle, organisationnelle comme nous l'avons évoqué précédemment qui met au défi les processus d'innovation.

Les systèmes d'aide et de soins à domicile retenus sont des cas contrastés, au sein de régimes institutionnels fédéraux. De façon schématique, le cas allemand fait l'objet d'un financement et d'une gouvernance centralisée - assurance sociale fédérale - et d'une mise en œuvre encadrée par des lois des Länder mais qui repose sur l'essentiel sur une logique de marché. En revanche en Suisse, une partie du financement et de la gouvernance intervient dans le contexte fédéral, mais les financements complémentaires nécessaires sont laissés à l'appréciation des cantons, qui, le plus souvent, délèguent cette tâche aux communes. La mise en œuvre communale est importante dans une grande majorité de cantons suisses. Les études de cas retenues à Ahlen en Allemagne et dans le canton de Vaud en Suisse, l'ont été pour leur caractère original dans leur contexte national respectif, mais surtout parce qu'elles présentent des profils d'innovation contrastés. À grands traits, le cas de Ahlen est un succès au plan du déploiement des mesures, mais un échec pour ce qui concerne le changement des pratiques alors que le cas vaudois est plutôt un succès sur ces deux aspects.

Le projet de Ahlen en Allemagne vise à favoriser l'accès des personnes issues de l'immigration aux services d'information, d'orientation, mais aussi d'aide et de soins à domicile pour les cas de démences, alors même que le maigre recours de cette population indique leur faible intégration dans les réseaux sanitaires et sociaux disponibles localement.

Sous sa forme actuelle, le projet des Alzamis est novateur dans le contexte vaudois, mais aussi suisse. Il propose en effet un service de répit à domicile dans le cas de personnes atteintes de la maladie d'Alzheimer ou de pathologies proches. Il comble ainsi un manque criant dans l'offre de service, met en évidence le travail des proches de même que les risques d'épuisement qui en découlent et propose une offre spécialisée et professionnelle dans un contexte où ce type de soin est renvoyé traditionnellement à la sphère informelle.

Nous avons conduit pour cette recherche des études de cas « en contexte », c'est-à-dire que nous avons systématiquement relié les cas locaux étudiés à leur contexte régional - du Land en Allemagne et du canton en Suisse -, mais aussi à leur contexte national. Des entretiens ont

\footnotetext{
${ }^{1}$ Ces deux projets ont été conduits par les auteur.e.s de cet article. Le premier (2011-2013) a été financé par la Mire-Drees et a donné lieu à une coopération entre le Centre Marc Bloch de Berlin CNRS, le Wissenschaftszentrum de Berlin et l'Université de Genève. Le second (2012-2015) a été financé par la Fondation Leenaards (Lausanne) et conduit depuis la Haute école de travail social de Genève, HETS-HES.SO et I'Institut d'Etudes de la citoyenneté (Incite) de l'Université de Genève, projet intitulé : "Apprentissage et innovation dans les réseaux d'aide et de soins à domicile- le cas des démences. Une comparaison Allemagne, Écosse et Suisse». (Lucas, Giraud, Sgier, Rüfenacht 2014).
} 
été conduits à l'échelle locale mais aussi auprès d'acteurs inscrits dans le contexte régional (canton ou Land) ou fédéral. Outre les initiateurs des innovations, nous avons interviewé les acteurs sociaux impliqués ou dont l'implication a été sollicitée, à un stade ou à un autre de la trajectoire d'innovation. Il peut alors s'agir d'acteurs ancrés au sein d'associations partenaires, concurrentes ou complémentaires, de services administratifs ou de pouvoirs publics, ou encore de représentants de communautés moins structurées au plan institutionnel. Ainsi, 11 acteurs ont été interviewés dans le canton de Vaud et 11 en Allemagne, entre mars et juillet 2013 (Lucas et al. 2014). Dans chacun des cas, nous avons procédé à des entretiens rétrospectifs et suggéré aux personnes interviewées de retracer la trajectoire de l'innovation depuis le moment où elles avaient eu à faire avec elle et dans quel contexte elles l'avaient reçue. Nous avons par ailleurs élargi la récolte systématique des données disponibles, notamment avec la littérature grise, et avons procédé au croisement des informations entre les différentes sources. Tous les entretiens conduits ont été retranscrits de façon à permettre une analyse des discours approfondie. Cette analyse a permis de repérer l'ouverture des discours d'innovation initiaux à des éléments d'autres discours dans le but de sceller des alliances discursives, mais aussi d'identifier les négociations portant sur les ressources. Nous avons spécifiquement travaillé trois étapes de la trajectoire d'institutionnalisation : la formulation originelle de l'innovation, son extension à des acteurs autres que les initiateurs de l'innovation au moyen de la conclusion d'alliances discursives et ses modalités d'inscription dans des cadres institutionnels.

\section{Mécanismes sociaux à l'œuvre dans les trajectoires d'innovation}

Comment les promoteurs d'une innovation parviennent-ils à favoriser son institutionnalisation? Comment les coalitions en soutien à ces innovations peuvent-elles se développer alors même que le domaine est fragmenté ? Nos cas empiriques renseignent sur la constitution des coalitions discursives portant sur les finalités et scellant les accords stratégiques sur les ressources dans l'institutionnalisation des innovations.

\subsection{Extension du discours sur les finalités : potentiels et limites}

Dans nos deux études de cas, la capacité des acteurs ayant initié l'innovation sociale à élargir la coalition des acteurs la soutenant s'avère contrastée. Dans le cas helvétique, l'innovation suscite des intérêts convergents de la part d'acteurs ancrés dans des espaces institutionnels divers. Sans céder aux valeurs qui l'ont inspirée, les objectifs de l'innovation évoluent dans le contexte de la construction de la coalition. Dans le cas allemand en revanche, les acteurs communaux qui portent l'innovation restent dans une relation plus hiérarchique que véritablement ouverte aux partenaires sollicités et ne parviennent ainsi pas à en faire des alliés actifs et mobilisés.

Les Alzamis : une reconnaissance des proches aidants dans les soins à domicile.

Le canton de Vaud est le premier canton, en Suisse, à s'être doté d'un programme cantonal Alzheimer en 2010 et, dans la foulée, à revendiquer une politique active de soutien aux proches de personnes en situation de dépendance. Le développement du projet des Alzamis est intimement lié cette dynamique politique. 
À la fin des années 1990, forte de son expérience personnelle, une habitante de Lausanne imagine un service de relève à domicile pour les familles. Le concept est relayé par l'association Alzheimer Vaud, qui identifie le manque de soutien adapté aux malades et à leurs familles comme une lacune du système de soin vaudois ${ }^{2}$. À l'instar de nombreuses associations du domaine du social ou de la santé, Alzheimer Vaud est alors une petite section composée principalement d'individus concernés à titre personnel par la maladie. Le premier narratif support de cette innovation s'articule autour de la spécificité de la maladie d'Alzheimer. Accompagner un membre de sa famille atteint d'Alzheimer est considéré comme une expérience particulière qu'il s'agit de reconnaître. Cette approche se double d'une vision du maintien à domicile centrée sur la famille, qui exclut toute prise en charge extérieure. Les prestations fournies par les acteurs du réseau sont d'ailleurs jugées inadaptées aux besoins des malades et de leurs proches. La spécificité de la maladie justifie toutefois la formation des intervenantes ainsi qu'une rémunération permettant de garantir leur engagement sur le long terme. Pour autant, cette première version de l'innovation reste inspirée par ce qu'Annie Dussuet (2005) a nommé un « modèle domestique » de l'aide à domicile, à savoir participant d'une logique de don, caractérisé par la flexibilité de ses prestations et rendu possible par une mise à disposition de femmes au service de leurs proches.

Avec la multiplication du nombre des prises en charge, le projet est rapidement confronté, sur le terrain, à des difficultés d'intégration horizontale. Le narratif déployé heurte les acteurs du réseau et le service des Alzamis est ignoré ou perçu comme concurrent. Dans ce contexte, les premières collaborations routinisées entre les Alzamis et les services d'aides à domicile restent limitées. L'élargissement de la coalition ne passe donc pas principalement par une association avec le réseau d'aide et de soin à domicile, mais plutôt par une alliance avec des acteurs du domaine sanitaire cantonal. Au milieu des années 2000 en effet, la coalition en soutien aux Alzamis s'étend à des acteurs clés du réseau cantonal, grâce à l'intégration, au sein du comité d'Alzheimer Vaud, de professionnels du domaine de la santé, de la psychogériatrie en particulier. Le contenu du projet n'est pas fondamentalement transformé par cet élargissement de la coalition, qui confirme la volonté de renforcer la formation des accompagnantes. En revanche, les éléments de discours critique vis-à-vis des acteurs du réseau de soins à domicile s'estompent au profit d'une approche en termes de complémentarité.

L'institutionnalisation des Alzamis quant à elle est rendue possible par l'implication du gouvernement cantonal et de son administration en faveur du projet, d'abord à travers des subventions à l'association Alzheimer Vaud puis par l'implication de l'association dans l'élaboration du plan cantonal Alzheimer et de la politique de soutien aux proches aidants, qui inclut les proches de personnes handicapées ou personnes âgées dépendantes. Le principe même sur lequel se fonde l'innovation, à savoir la reconnaissance des proches et la nécessaire spécialisation, inspirent même la nouvelle politique des proches aidants. Ce processus aboutit à une institutionnalisation des Alzamis en tant que service de relève spécialisé, reconnu, financé

\footnotetext{
${ }^{2}$ Significativement, le projet se développe alors que se met en place, au niveau fédéral en Suisse, la nouvelle loi sur l'assurance maladie obligatoire, LaMAL, entrée en vigueur en 1996. Parmi les conséquences de cette entrée en vigueur, un nouveau système de tarification des soins, qui exclut du remboursement toute prise en charge non médicalisée ou ne relevant pas d'une liste restreinte de " soins de base ». Dans ce contexte, le temps de présence, de discussion ou d'accompagnement d'une personne atteinte de démence ne peut plus être comptabilisé dans un forfait de soin.
} 
et contrôlé par le canton, avec pour objectif de « prendre le relais des proches des malades et de favoriser le maintien à domicile ». Son déploiement sera toutefois limité par les réticences des proches à demander de l'aide, un comportement de non-recours aux « aides formelles » socialement ancré dans un modèle de care helvétique centré sur la famille et surtout, sur les normes de sollicitude qui s'imposent encore fortement aux épouses et aux filles ${ }^{3}$.

L'intégration des Alzamis dans ces deux nouveaux référentiels de politiques publiques (politique Alzheimer et soutien aux proches aidants) contribue à faire évoluer le narratif de l'innovation. En premier lieu, la collaboration entre prestataires des soins à domicile est valorisée, s'inscrivant désormais dans des conventions entre l'organisation faîtière des soins à domicile et l'association Alzheimer Vaud. Par ailleurs, la focale élargie - du cas des démences à la réflexion cantonale transversale sur les proches aidants - tend à dissoudre l'ancrage domestique du projet dans une vision dénaturalisée et défamilialisée des proches. Enfin, d'une réponse associative à une lacune du système se déployant dans les espaces sociaux ignorés des gestionnaires de santé, les Alzamis se conçoivent aujourd'hui comme un instrument au service d'une politique basée sur la mobilisation des ressources communautaires.

\section{Ahlen et la coordination des politiques sociales au bénéfice des personnes issues de l'immigration} atteintes de démence.

Ahlen est une commune d'une région industrielle allemande qui a développé de longue date une politique de l'intervention sociale fondée sur l'intégration des acteurs locaux publics et privés présents sur son territoire. Cette commune a posé le constat au début des années $2000 \mathrm{du}$ faible recours des personnes issues de l'immigration, notamment turque, aux services sociaux destinés au grand âge, en particulier pour ce qui touche aux démences. La commune a ainsi entrepris un travail de coordination des services sociaux et des politiques de la ville, mais aussi d'ouverture active en direction des associations et réseaux communautaires de manière à lutter contre ce trop faible recours.

Le premier narratif qui a structuré la politique initiée en direction des personnes issues de l'immigration par la commune de Ahlen a porté sur la réussite de la politique d'intégration des services et, très largement, de l'offre d'intervention sociale, saisie dans sa globalité, orchestrée de longue date par la ville de Ahlen. Le déficit est apparu particulièrement important alors que les effets des politiques d'information et de soutien vis-à-vis des personnes atteintes de démences et de leurs proches se sont avérés importants. L'absence des personnes issues de l'immigration a été comprise comme un défaut de communication entre les réseaux de la communauté turque et les réseaux locaux d'action publique, notamment sanitaires et sociaux. Les acteurs municipaux ont alors principalement formulé les besoins d'intervention, précisément en termes de réseaux. Au-delà des mots d'ordre visant à adapter les politiques des démences aux besoins de la communauté turque, les priorités étaient ciblées vers une double «intégration». En premier lieu, il s'agissait de mieux intégrer les politiques sociales et les politiques de la ville qui comptent les personnes issues de l'immigration parmi leurs destinataires. En second lieu, il fallait mieux intégrer les réseaux sanitaires et sociaux avec les réseaux communautaires, spécialement, ceux des populations turques ou d'origine turque.

\footnotetext{
${ }^{3}$ Sur le régime de genre en Suisse voir Giraud \& Lucas (2014). Sur le non-recours aux aides financières à l'échelle cantonale en Suisse, voir Lucas et al. (2018) et Hümbelin (2016).
} 
Les coalitions qui se sont formées dans le cadre de cette politique ont été plutôt couronnées de succès. L'intégration des politiques sanitaires et sociales vers celles de la ville avait pour but de se rapprocher des quartiers de résidence des personnes et d'ainsi plus facilement irriguer dans les réseaux communautaires. Cette première démarche a fonctionné mais a impliqué d'ajouter du contenu au discours porteur de l'innovation. Aux discours d'information sur la maladie, la prévention, les services disponibles et les réseaux d'aide et de soins ont été incorporés des messages sur l'adaptation des logements, les services de proximité, et la complémentarité des mesures entre ville, social et sanitaire. À la suite de cette coordination renforcée des politiques et services relevant de la sphère d'influence de la ville, le rapprochement des associations et réseaux communautaires est intervenu, après un temps de scepticisme et attentisme réciproques. Cependant, ce second rapprochement n'a pas débouché sur une véritable «coalition » au sens de M. Hajer notamment parce que le discours d'innovation ne s'est pas ouvert à des contenus en provenance de ces associations ou réseaux communautaires. Sur le papier, ces réseaux ont accepté de relayer les informations diffusées depuis les réseaux municipaux et para-municipaux, mais ils ne se sont pas approprié le contenu des messages et n'ont pas contribué à les alimenter.

Dans ce contexte de coalition discursive inachevée, l'institutionnalisation de l'innovation sociale est restée en demi-teinte. Si les actes de mise en œuvre programmés ont bien été réalisés - séances d'information, recrutement de personnes dédiées à l'accompagnement de personnes issues de l'immigration et atteintes de démences, etc. -, les échos auprès de la population des destinataires ultimes sont restés faibles. Le constat d'échec est de ce point de vue partagé par tous les acteurs. L'institutionnalisation, au sens de la création d'un dispositif, a débouché sur des résultats, mais la lutte contre le non-recours des personnes âgées issues de l'immigration turque aux dispositifs d'information et de soutien dans le champ de la politique de prise en charge des démences reste un échec. Le fait qu'aucun élément de discours en provenance des réseaux communautaires n'ait été intégré au discours porteur de l'innovation a obéré la mobilisation effective de ces derniers.

Dans le cas suisse, la conclusion de coalitions avec des acteurs éloignés des initiants directs de l'innovation sociale débouche sur une transformation significative du discours de l'innovation mais qui ne remet pas fondamentalement en cause ses valeurs principales. Ainsi, la conception, centrale pour l'innovation, de la figure du proche aidant donne lieu à une ouverture importante alors que celle du soin à domicile se trouve profondément modifiée. Cependant, toutes deux restent des valeurs essentielles autour desquelles se conçoit le « bon soin » aux personnes atteintes de démence. En dépit de la fragmentation du réseau, cette évolution du narratif de l'innovation permet, dans le cas vaudois un élargissement significatif de la coalition qui soutien l'innovation et favorise sa mise en œuvre. Qui plus est, on assiste à un enrichissement mutuel puisqu'une partie du narratif des Alzamis est reprise par les acteurs du réseau.

En revanche, dans le cas allemand, la coalition avec les services municipaux et les réseaux des politiques de la ville a sans doute permis d'intégrer des préoccupations et d'étendre le périmètre d'action en matière d'information et d'orientation dans le cas des démences. Cependant, une véritable ouverture du discours de l'innovation aurait surtout pu être acquise par le biais de l'intégration de contenus et valeurs en provenance des acteurs et réseaux issus de l'immigration, ce qui n'a pas été le cas. On peut faire l'hypothèse que la faible effectivité de cet aspect des coalitions d'acteurs n'a pas permis dans le cas local allemand de mobiliser 
véritablement les destinataires ciblés par l'ensemble de la démarche d'innovation.

\subsection{Mécanismes de mobilisation des complémentarités entre ressources}

Nos deux études de cas confirment que la diffusion d'une innovation et sa traduction dans un changement social significatif, implique pour une part que soient conclus des accords sur les finalités et valeurs porteuses de l'innovation. Qu'en est-il à présent des aspects plus instrumentaux? Quels sont les mécanismes sociaux, les types d'accord, les formes d'interaction entre acteurs qui permettent que les ressources nécessaires à la réalisation de l'innovation sociale soient mobilisées de façon effective?

\section{Les Alzamis : l'intégration cantonale comme stratégie gagnante}

De façon sans doute plus marquée que dans de nombreux autres cantons suisses, la politique d'aide et de soin à domicile du canton de Vaud est du ressort du canton et fait l'objet d'une politique volontariste, laissant une moindre marge de manœuvre aux communes. Toutefois, une part importante de la gouvernance de l'aide et des soins à domicile se joue concrètement au niveau régional (plusieurs communes).

En 2008, une importante réorganisation des échelles de responsabilité et des financements entre les niveaux territoriaux en Suisse entre en vigueur. Elle marque la fin de la subvention fédérale de nombreux projets dans le domaine de l'aide à domicile notamment, dont le financement est transféré aux cantons ou aux communes. ${ }^{4}$ La reprise des financements n'est pas automatique et dépend des configurations de pouvoir cantonales, comme nous avons pu l'observer dans le cas du handicap (Giraud, Lucas 2007). Par ailleurs, les Alzamis ne peuvent plus compter sur le financement d'Alzheimer Suisse. Au début des années 2000 en effet, l'expansion du projet a généré une crise financière avec l'organisation nationale, qui répondait alors des finances des sections cantonales. Depuis, les membres des sections cantonales paient leurs cotisations à l'association fédérale, et les sections locales sont libres de chercher des fonds auprès d'acteurs institutionnels. La question se pose alors de savoir si les sections doivent s'engager dans des prestations d'aide et de soins à domicile, en dehors de leurs traditionnels groupes d'entraide.

Dans ce contexte, et à partir de 2007, on assiste à une institutionnalisation cantonale du service Alzamis. L'innovation bénéficie alors d'une véritable « fenêtre d'opportunité » politique. En effet, plusieurs réformes parallèles sont lancées dans le canton en lien avec la législature 2007-2012, contribuant à positionner le canton comme un précurseur en matière de politique Alzheimer dans le contexte national ${ }^{5}$. Dans ce cadre, la participation de l'Association Alzheimer Vaud, associée à l'élaboration de ces programmes, accélère et amplifie le processus de reconnaissance cantonale du service de relève des Alzamis. Les autorités reprennent la subvention des Alzamis, en y mettant des conditions, liées notamment au caractère professionnel de sa gestion.

Ce choix de positionner la section cantonale - à travers son projet Alzamis- comme un

${ }^{4}$ Réforme de la péréquation financière et de la répartition des tâches entre la Confédération et les cantons (RPT).

${ }^{5}$ Centralisation cantonale de l'aide et des soins à domicile, plan cantonal Alzheimer relevant de la politique en santé mentale; politique des proches aidants, relevant du Service des assurances sociales et de I'hébergement; et, à un niveau plus large, la formulation d'un nouveau concept de politique en matière de vieillissement, le Programme «Vieillissement et santé ». 
prestataire du réseau d'aide et de soin à domicile, dans le cadre de la nouvelle politique qui se met en place, relève d'une stratégie de la section cantonale. Celle-ci se distingue alors de celle d'Alzheimer Suisse, pour qui la mobilisation politique apparaît prioritaire. La stratégie de la section vaudoise se révèle gagnante à plusieurs titres. En premier lieu, pour la section, les Alzamis représentent clairement une ressource stratégique :

"La chance qu'on a, c'est d'être reconnu par l'État, si on n'avait pas ça, je pense qu'on serait une petite section, si on n'avait pas ce service Alzamis, on serait une petite section, comme il y en a certaine, qui ont une permanence téléphonique un jour par semaine et puis une boîte aux lettres chez un membre du comité.»

Par ailleurs, le recours au niveau cantonal permet au projet innovant des Alzamis de contourner pour partie les blocages liés aux difficultés d'intégration horizontale. En effet, dans le cadre du plan Alzheimer, le canton donne de claires impulsions en vue d'un renforcement des coopérations entre les Alzamis et les services régionaux d'aides et de soins à domicile ${ }^{6}$. Cette incitation à la coopération, dans une perspective top-down, produit des effets certains sur le réseau et est considérée par de nombreux acteurs comme un élément déterminant dans le succès de la coopération. Enfin, l'institutionnalisation du projet Alzamis va de pair avec la volonté du canton d'augmenter le nombre des bénéficiaires de ce service. L'association Alzheimer Vaud a ainsi bénéficié de subventions significatives pour une campagne de promotion des Alzamis, visant à lever la réticence des familles à demander de l'aide.

\section{Ahlen entre opportunisme et émancipation vis-à-vis des acteurs centraux}

Dans le cas de Ahlen et de son projet municipal d'intégration des réseaux prestataires et de ceux des personnes issues de l'immigration turque, la mobilisation des ressources de mise en œuvre est marquée par un double jeu. En premier lieu, les communes qui étaient des actrices majeures du domaine de l'aide et des soins à domicile, ont été fortement affaiblies par les réformes des années 1990. En Allemagne, la régulation du domaine de l'aide et des soins à domicile est localisée à l'échelon fédéral, même si les Länder ont la responsabilité du secteur sanitaire et doivent se doter de lois dans le domaine. On retrouve un équilibre pratiquement similaire pour ce qui touche au financement des acteurs sociaux sanitaires

. Les normes du financement sont définies dans l'arène fédérale, mais les caisses qui organisent les flux financiers opérationnels sont organisées à l'échelle des Länder. Relativement démunies du point de vue du pouvoir de régulation et des finances, les communes qui avaient l'habitude de jouer un rôle central dans le domaine du sanitaire et du social et conservent une grande maîtrise des réseaux d'acteurs locaux et régionaux - le cas de Ahlen est plus que paradigmatique en la matière - ont dans bien des cas cherché à récupérer un rôle central dans le domaine (Giraud, 2017).

La mobilisation de l'action publique sur des enjeux émergents comme celui des cas complexes (les démences par exemple à partir des années 2000) a constitué une opportunité de rebattre les cartes des relations entre les échelles d'action publique. Cette époque a également été en Allemagne comme ailleurs celle de l'introduction des financements par

\footnotetext{
${ }^{6}$ Ainsi, par exemple, des personnes ressources sont nommées dans les 52 centres médicaux-sociaux du canton afin de mieux identifier les situations liées aux démences et d'en référer, le cas échéant, aux Alzamis. L'évaluation de la prestation des Alzamis peut désormais se faire de manière autonome par les coordinatrices régionales du service et une délégation des soins de base aux accompagnatrices Alzamis été formalisée.
} 
appel d'offres qui a introduit de nouveaux rapports de pouvoirs entre acteurs institutionnels.

En second lieu, la stratégie des acteurs porteurs d'innovation s'est repositionnée dans ce contexte renouvelé. La commune de Ahlen a développé, dès le début des années 2000 , une véritable capacité de mobilisation de ressources financières en remportant un grand nombre d'appels d'offres et de prix à l'innovation. Loin d'être contrainte par ces dispositifs, Ahlen a gagné des marges d'action déterminantes pour mettre en œuvre sa politique de programmation et planification et assurer sa position de pouvoir en dépit de la déstabilisation institutionnelle des communes allemandes dans le secteur.

En revanche, la ville ne s'est pas inscrite dans les principaux programmes de mobilisation des acteurs locaux au profit de la prise en charge de la santé des migrants vieillissants qui avaient été délégués par le puissant Land de Rhénanie du Nord Westphalie (près de 18 millions d'habitants) à l'association nationale traditionnellement en charge des politiques sociales auprès des migrants issus de Turquie, l'ArbeiterWohlfahrt. Dès les années 1990, le Land a en effet délégué au profit d'une des principales associations de mise en œuvre des politiques sanitaires et sociales d'Allemagne (Arbeiterwohlfahrt) d'importants moyens visant à réduire le non-recours des migrants âgés aux politiques sociales. Une certaine expérience ainsi que du matériel de sensibilisation et d'information - notamment en langue turque - à propos de la maladie d'Alzheimer et des démences était disponible auprès de cette association parapublique. Ahlen n'a jamais eu recours à cette offre difficilement intégrable dans le « système de Ahlen » au centre duquel se trouve la commune elle-même. La commune s'est en revanche directement saisie de ressources mises à disposition par le Land sous la forme d'un poste destiné au maillage territorial dans le champ des politiques Alzheimer et dont la disponibilité était plus grande et intégrable presque directement dans le programme de la commune d'intégration et de mobilisation en faveur des personnes d'origine étrangère. En résumé, le succès remporté par la municipalité à des appels d'offres fédéraux (Demenzfreundliche Stadt) a permis aux acteurs locaux de dépasser les actions entreprises par des acteurs parapublics régionaux appuyés par le gouvernement du Land de Rhénanie du Nord-Westphalie spécifiquement en faveur de l'inclusion des migrants vieillissants dans les dispositifs de prévention et d'accompagnement des malades d'Alzheimer et maladies apparentées.

Ainsi, nos deux cas mettent en évidence l'importance de considérer, en plus des mécanismes discursifs mettant en jeu des valeurs, les relations stratégiques développées par les initiateurs d'une innovation, relations qui participent de la formulation commune des modalités de mise en œuvre. Les acteurs de l'innovation composent les ressources en fonction de leurs besoins, mais aussi des contreparties attendues. Dans le cas helvétique, on a pratiquement à faire à une relation d'échange entre un canton qui a besoin d'animer un domaine d'action publique dont l'importance est grandissante et une structure faiblement structurée à l'origine qui gagne une grande stabilité grâce au soutien cantonal décidé que lui accorde l'État de Vaud. Dans le cas allemand, l'attitude de la commune de Ahlen se révèle à la frontière de l'opportunisme. La ville choisissant avec beaucoup de liberté de se positionner sur les ressources en provenance de l'État fédéral ou du Land qui ne mettent pas au défi sa position de centralité et l'indépendance de ses choix dans le déploiement de sa stratégie. Dans cette perspective, la fragmentation du domaine apparaît autant comme une structure d'opportunité que comme un ensemble de contraintes. Si dans le cas suisse, les relations d'échanges entre les échelles d'action publique semblent relativement équilibrées 
et, là aussi permettent de contourner les fragmentations de façon efficace, le cas allemand montre une stratégie d'usage unilatéral des ressources du Land et du Bund par la commune, dans le but de conserver une forte autonomie politique. De ce point de vue, les complémentarités entre les capacités de ressources mobilisables à différents niveaux institutionnels ne se déclenchent pas dans le cas allemand de la même façon que dans le cas suisse.

\section{Conclusion : les diverses rationalités de l'innovation sociale}

La fragmentation de l'action publique, parce qu'elle introduit potentiellement des segmentations et des concurrences supplémentaires entre acteurs, est le plus souvent envisagée comme un obstacle rédhibitoire au changement, ou à l'innovation sociale. Les deux cas concrets analysés montrent que des mécanismes sociaux spécifiques permettent de surmonter ces facteurs de blocages des trajectoires de changement. Ces mécanismes ont trait à la capacité des porteurs de l'innovation à construire des coalitions discursives sur différents enjeux de l'action publique : d'une part sur les objectifs et valeurs de l'innovation, et d'autre part sur les ressources qui doivent être mobilisées et qui sont souvent localisées à des échelles d'action publiques spécifiques. Dans la poursuite de cet objectif, deux types de rationalités, qui sont le plus souvent opposées, se dégagent : une rationalité en valeur d'une part et une rationalité instrumentale d'autre part. Or, c'est bien de la capacité des porteurs de l'innovation sociale de mobiliser conjointement ces deux types de rationalités que se joue le destin des projets innovants.

Dans un article précédent, nous avons montré à quel point les objectifs initiaux d'une innovation dans le domaine des soins de longue durée, qui, conformément à la définition de l'innovation sociale, relèvent d'un registre de valeurs inspiré par la perspective d'une amélioration des services et prestations du point de vue des bénéficiaires, sont soumis aux fourches caudines d'une rationalité libérale qui peut se révéler fatale aux objectifs initiaux. Un processus que nous avions qualifié de «normalisation » de l'innovation. (Giraud et al. 2014). Dans cette perspective, un répertoire de valeur succède à un autre en fonction des arènes institutionnelles au sein desquelles circule l'innovation sociale. L'analyse approfondie de deux cas empiriques présentée dans le présent article confirme la présence de différentes rationalités à l'œuvre dans le processus d'innovation sociale, mais s'intéresse cette fois aux mécanismes évitant le risque de normalisation, tout en permettant le déploiement effectif de l'innovation.

Ce qui apparaît comme déterminant de ce point de vue, c'est l'extension au moins partielle du discours des objectifs de l'innovation à des éléments discursifs portés par les acteurs qui rejoignent la coalition. Pour cela, on constate une modification du sens des éléments discursifs qui forment le discours - mécanisme permettant précisément la construction d'un discours hégémonique (Howarth 2000). Ce sont ici des enjeux en valeurs qui servent de support à la narration commune rendant l'accord possible, comme l'illustre le cas des Alzamis.

Mais les porteurs de l'innovation doivent aussi garantir que le discours innovant pourra s'incarner tant dans des institutions que dans des pratiques sociales. De ce point de vue, la rationalité en valeur se révèle insuffisante à produire des effets concrets. En effet, la coordination entre acteurs dans la mobilisation des ressources relève d'une rationalité différente. Nos études de cas confirment que les ressources de l'action publique étant le plus souvent 
ancrées à des échelles d'action publiques différentes, les logiques de réciprocité ou au moins d'équilibre entre les acteurs localisés au sein de ces espaces différenciés, supposent la conclusion de négociations ou d'accords équilibrés entre eux. Ainsi, bien que fortement contraints par les contextes structurels de répartition des ressources, les promoteurs de l'innovation dans le canton de Vaud en Suisse et dans la commune de Ahlen, en Allemagne, développent des relations d'échange qui peuvent s'avérer payantes. De ce point de vue, la fragmentation du domaine apparaît même comme une opportunité, en ce qu'elle permet d'élargir les possibilités d'échanges entre échelles d'action publique.

En conclusion, l'institutionnalisation d'une innovation sociale, comme nouvel instrument d'action publique, suppose que ses deux logiques de fonctionnement, en valeur et instrumentale, soient concernées. Du côté de la rationalité en valeur, la mobilisation réelle des acteurs nécessite de nouer des compromis portant sur les objectifs qualitatifs de l'innovation sociale. L'extension ou la reformulation des objectifs affichés initialement par les porteurs de l'innovation n'implique pas nécessairement leur dénaturation. Du côté de la rationalité instrumentale, la mobilisation des ressources nécessaire à la mise en œuvre de l'innovation suppose également la conclusion d'accords avec des partenaires autonomes. Il s'agit alors d'ententes stratégiques fondées sur les complémentarités entre les positions de pouvoir, les échanges de ressources, et des accords bien compris ne portant pas au premier chef sur les objectifs. Ces deux logiques d'accords renvoient à deux acceptions fondamentales de la politique. La première concerne la définition des projets ou objectifs communs. La seconde porte sur les complémentarités productives entre les membres d'une communauté. 


\section{Bibliographie}

Bezès P. et Le Lidec P., 2016. Politiques de l'organisation - Les nouvelles divisions du travail étatique. Revue Française de Science Politique 66 (3), 407-433.

Benz A., 2009. Politik in Mehrebenensystemen. VS Verlag für Sozialwissenschaften, Wiesbaden.

BEPA, 2010. Empowering People, Driving Change : Social Innovation in the European Union. European Union / Bureau of European Policy Advisers, Luxembourg.

Duran C, 1999, 2010. Penser l'action publique. LGDJ, Paris.

Dussuet A., 2005. Tauvaux de femmes. Enquêtes sur les services à domicile. L'Harmattan, Paris.

Evers A. and Benjamin E., 2013. How to Approach Social Innovation. Lessons from Berlin. Paper presented at the $1^{\text {st }}$ International Conference on Public Policy, June 26-28, 2013, Grenoble, June 26-28.

Giraud O., 2017. L'introuvable démocratie du care? La gouvernance multiscalaire des systèmes d'aide et de soins à domicile des personnes âgées en Europe entre néo-familialisme et privatisation : les cas de Hambourg et d'Edimbourg. Revue Européenne des sciences sociales 55, 122-147.

Giraud O. et Lucas B., 2015. Overcoming Privacy Preserving Federalism - A Multiscalar Approach to the German and Swiss Shifts in Gender Regimes ». Published 1st July 2015. Fédéralisme Régionalisme 14

http://popups.ulg.ac.be/1374-3864/index.php?id=1392

Giraud O. et Lucas B., 2015. L'émergence d'une nouvelle catégorie d'action publique - le rôle des instruments dans la construction des « proches aidants ». Congrès de l'AFSP, Aix-en-Provence.

Giraud O., Lucas B., Falk K., Kümpers S. et Lechevalier A., 2014. Innovations in Local Domiciliary Long-Term Care : From Libertarian Criticism to Normalisation. Social Policy and Society 3 (3), 433-444

Giraud O. et Lucas B., 2007. Jeux d'échelles et référentiels d'action publique : la cantonalisation des politiques du handicap en Suisse In Faure et al. (éd.). L'action publique et les changements d'échelles : les nouvelles focales du politique. L'Harmattan, Paris, 147-166

Hajer M. A., 1997. The Politics of Environmental Discourse. Clarendon Press, Oxford.

Halpern C., Lascoumes P. et Le Galès P., 2014. Introduction / L'instrumentation et ses effets débats et mises en perspective théoriques In Halpern C., Lascoumes P. et Le Galès P L'instrumentation de l'action publique. Controverses, résistance, effets. Presses de Sciences Po, Paris, 15-62.

Hooghe L., Marks G., 2003. Unraveling the Central State, but How? Types of Multi-level Governance. American Political Science Review 97 (2), 233-243.

Howaldt J., Schwarz M., 2010). Social Innovation - Concepts, research Fields and International Trends. Sozialforschungsstelle, Dortmund.

Howarth D., 2000. Discourse. Open University Press, Buckingham.

Hümbelin O., 2016 Nichtbezug von Sozialhilfe und die Bedeutung von regionalen Unterschieden. Working Paper No. 21.University of Bern Social Sciences, Bern.

Jenson J., Harrisson D., 2013. Social innovation research in the European Union : approaches, findings and future directions. Policy Review. European Commission, Brussels.

Le Bihan-Youinou B., 2011. La généralisation des «prestations monétaires » en Europe. Quel impact sur l'organisation de l'aide quotidienne aux personnes âgées en perte d'autonomie? Gérontologie et société $138,83-97$

Lucas B.,Ludwig, C., Chapuis, J., Maggi, J. et Crettat,E., 2018. Le non-recours aux prestations sociales à Genève. Quelles adaptations de la protection sociale aux attentes des familles en situation de précarité? Haute école de Travail social, HES-SO, Genève.

Lucas B., Giraud O., Sgier L. et Rüfenacht D., 2014. Apprentissage et innovation dans les réseaux d'aide et de soins à domicile - le cas des démences : une comparaison Suisse, Allemagne, Écosse. Rapport de recherche. Resop-Université de Genève, Fondation Leenards, Lausanne. 
Lucas B., Giraud O., Galleguillos E. et Maggi J., 2010. Politiques d'aide aux malades Alzheimer en Suisse. Définition des besoins et gestion des conflits à chaque étape du parcours de vie Vol. 2. Resop, Département de science politique, Université de Genève, Genève.

Rose R. and Davies Ph., 1994. Inheritance in Public Policy. Change without Choice in Britain. Yale University Press, New Haven,

Sinclair S., Baglioni S., 2014. Review Article : Social Innovation and Social Policy - Promises and Risks. Social Policy \& Society 13 (3), 469-476.

Streck W., Thelen K. (eds), 2005. Beyond Continuity. Institutional Change in Advanced Political Economies. Oxford University Press, Oxford.

Varone F., Nahrath S., Aubin D. et Gerber J.-D., 2013. Functional Regulatory Spaces. Policy Sciences 46 (4), 311-333.

Varone F., Bonvin J.-M. (eds), 2004 Regards croisés sur la Nouvelle Gestion Publique. Les Politiques Sociales $64(1-2), 4-17$. 